\title{
Novel cardiovascular risk biomarkers in carotid atherogenesis
}

\author{
Federico Carbone ${ }^{1}$ \& Fabrizio Montecucco*,1,2 \\ ${ }^{1}$ First Clinic of Internal Medicine, Department of Internal Medicine, University of Genoa, 6 viale Benedetto XV, 16132 Genoa, Italy \\ ${ }^{2}$ IRCCS Ospedale Policlinico San Martino, Genova 10 Largo Benzi, 16132, Genoa, Italy \& Center of Excellence for Biomedical \\ Research (CEBR), University of Genoa, 6 viale Benedetto XV, 16132 Genoa, Italy \\ *Author for correspondence: Tel.: +39 01035332 204; Fax: +39 010353 8686; fabrizio.montecucco@unige.it
“ When compared with controls, SOST was able to reduce matrix degradation and inflammatory cell infiltration, thus resulting by the suppression of the $\mathrm{Wnt} / \beta$-catenin responsive genes”

First draft submitted: 6 June 2018; Accepted for publication: 24 July 2018; Published online: 19 September 2018

\section{Keywords: biomarker $\bullet$ cardiovascular risk • carotid atherosclerosis • obesity • OPN • SOST}

The identification and validation of biomarkers of atherosclerotic plaque progression and vulnerability represent an urgent need in preventive cardiology. Among different classes of biomarkers, the study of bone mediators might be a very promising field of research. On the one hand, they can contribute to determine the degree of calcification and then the number of interfaces between rigid and distensible portions of the plaque exposed to the shear stress forces of the blood flow. In addition, these molecules are also emerging as mediators of plaque inflammation. Especially the axis involving the RANKL and OPG has been demonstrated to influence plaque stability by interfering with the complex balance between anti- and pro-inflammatory molecules [1]. More specifically, RANKL dose-dependently induces neutrophil degranulation in vitro, in addition to positively correlating with serum levels of neutrophil proteases in subjects with coronary calcifications [2]. Conversely, the role of two other mediators of atherosclerotic plaque calcification, such as osteopontin OPN and SOST, still remains unclear.

OPN is a secreted protein that plays a role in both physiological and pathological processes. An increase in the circulating levels of OPN has been observed in many acute and chronic inflammatory diseases [3,4]. Within the vascular system, OPN appears to be an important regulator of vascular calcification, being associated with mineralized deposits in both human and mouse models. OPN is highly expressed in human and murine atherosclerotic lesions, where it co-localizes with macrophages and foam cells. Not surprisingly OPN was recently demonstrated as a strong macrophage activator, acting as a promoter of their migration, prolonged survival and foam cell generation [5]. The OPN network also includes a potential upregulation of other proinflammatory mediators involved in the atherosclerotic process, (i.e., angiotensin II and oxidized low-density lipoproteins).

Furthermore, there is accumulating evidence that OPN may be weakened by treatments reducing atherosclerosis [6]. Despite this growing body of results, whether OPN may be useful for a better cardiovascular (CV) risk stratification has not yet been established. Our research group recently reported that serum levels of OPN predicted the occurrence of adverse CV events in patients with severe carotid atherosclerosis undergoing endarterectomy [7]. Such data confirmed previous high-powered clinical trials. Zwakenberg and colleagues recently identified serum OPN as a biomarker strongly associated with CV risk in a cohort of patients with Type 2 diabetes [8]. Nevertheless, those observations raise some complexity about OPN system, starting from its main source of production. Different research groups reported that OPN is also largely secreted by macrophages recruited into the dysfunctional adipose tissue $[9,10]$. In this regard, a role of aging in determining visceral adipose tissue dysfunction with associated increase in OPN production and release has been described [10]. It is therefore conceivable that both aging as well as some obesity phenotypes (i.e., those associated with higher cardio-metabolic risk) could share pathophysiological signs of dysfunctional adipose tissue, including the upregulated synthesis of OPN. More recently, different mediators have been suggested as a further link between dysfunctional adipose tissue and development of carotid atherosclerosis. 
Among the most promising molecules, especially pigment epithelium-derived factor and lipopolysaccharide-binding protein are emerging as potential markers of obesity-related insulin resistance [11,12].

According with those evidences, we also reported that circulating levels of OPN significantly correlated with histopathological features of plaque vulnerability, such as an increased intraplaque content of MMP-9, neutrophils and proinflammatory M1 macrophage subset [7]. Still some questions about the real biological activity of OPN within atherosclerotic plaques remain unanswered. Intraplaque OPN may actively promote neutrophil migration and then their recruitment from the blood stream [13]. Similarly, a modulatory role of OPN on monocyte/macrophage polarization has also been described [14]. Although many efforts are required to clarify the underlying molecular mechanisms, OPN gene expression upregulation has been associated with higher uptake of (18)F-FDG in a PET study on murine atherosclerosis [15]. The recent development of an OPN-specific MRI probe is expected to potentially improve current knowledge about the role of OPN in atherosclerosis [16].

As a product of the Sost gene, SOST is a 190-aminoacids glycoprotein acting as a negative regulator of bone formation. More specifically, the binding of sclerostin to the low-density lipoprotein receptor-related proteins- 5 and -6 , inhibits the Wnt/ $\beta$-catenin pathway. As a result, SOST was shown to suppressed the transcription of anabolic genes involved in osteoblast/osteocyte activity. However, the Sost gene is widespread expressed in the body, with markedly upregulated levels within the heart, aorta, kidney and lung [17]. Several studies demonstrated a relevant role for $\mathrm{Wnt} / \beta$-catenin in atherosclerosis, through a complex signaling pathway that also can control the downstream expression of OPG, OPN and several MMPs. It has been then hypothesized that SOST may also have a role in atherosclerosis. However, the association between SOST and arterial wall calcification remains unclear. Similarly, the contribution of SOST to atherosclerotic plaque development and progression has not yet been established. In the early stages of the disease, both negative and positive correlations have been reported with carotid intima media thickness and arterial stiffness, respectively $[18,19]$. Clinical studies also showed a positive association between circulating SOST levels and atherosclerotic burden [20] and plaque vulnerability [21], but the underlying mechanisms are still unknown. Experimental studies reported some paradoxical anti-inflammatory properties of SOST. Indeed, both Sost transgenic mice and recombinant sclerostin administration were able to inhibit aortic aneurysm formation and atherosclerotic plaque development in $A p o E^{-/-}$mice [22]. When compared with controls, SOST was able to reduce matrix degradation and inflammatory cell infiltration, thus resulting by the suppression of the $\mathrm{Wnt} / \beta$-catenin responsive genes (i.e., $O P G, O P N$ and $M M P-9$ ). These results highlight an apparent contrast between clinical and experimental findings. In addition, this aspect strongly emphasizes a gap of knowledge in the molecular pathways triggered by SOST in atherosclerotic inflammation. Wnt signaling has been largely described as a regulator of the dysmetabolic milieu underlying atherosclerosis. Wnt signaling induces endothelial cell proliferation, survival, as well as the enhancement of monocyte adhesion and transendothelial migration [23]. Wnt signaling activation has also been implicated in the proinflammatory macrophage polarization and foam cell generation as well as in the smooth muscle cell migration and proliferation [23]. Although surprisingly, the positive association observed between atherosclerotic burden and serum levels of SOST (a Wnt signaling pathway inhibitor) might be a defensive mechanism to delay atherosclerotic progression. However, further studies are needed to address many questions about the role of SOST in atherosclerosis. As first, it is mandatory to identify whether SOST is locally synthetized with atherosclerotic plaques and which cell/tissue could be its main source of production. Furthermore, the identification of target cells will pave the way for investigating potential therapeutic approaches, also considering that two anti-SOST antibodies (i.e., romosozumab and blosozumab) have been recently approved for clinical use against osteoporosis. In conclusion, the role of those mediators in carotid atherosclerosis and its acute ischemic complications, such as ischemic stroke, is unclear and requires additional clinical and basic research studies. Conversely, these molecules may have a role as predictors of successful bariatric/metabolic surgery [24]. Additionally, the investigation of selective treatments and pathophysiological pathways triggered by these two molecules might provide in the next future promising therapeutic strategies to reduce carotid atherogenesis.

Financial \& competing interests disclosure

The authors have no relevant affiliations or financial involvement with any organization or entity with a financial interest in or financial conflict with the subject matter or materials discussed in the manuscript. This includes employment, consultancies, honoraria, stock ownership or options, expert testimony, grants or patents received or pending, or royalties.

No writing assistance was utilized in the production of this manuscript. 


\section{References}

1 Quercioli A, Montecucco F, Bertolotto $\mathrm{M}$ et al. Coronary artery calcification and cardiovascular risk: the role of RANKL/OPG signalling. Eur. J. Clin. Invest. 40(7), 645-654 (2010).

2 Quercioli A, Mach F, Bertolotto M et al. Receptor activator of NF- kappaB ligand (RANKL) increases the release of neutrophil products associated with coronary vulnerability. Thromb. Haemost. 107(1), 124-139 (2012).

3 Morimoto J, Kon S, Matsui Y, Uede T. Osteopontin; as a target molecule for the treatment of inflammatory diseases. Curr. Drug Targets 11(4), 494-505 (2010).

4 Carbone F, Vuilleumier N, Burger F et al. Serum osteopontin levels are upregulated and predict disability after an ischaemic stroke. Eur. J. Clin. Invest. 45(6), 579-586 (2015).

5 Wolak T. Osteopontin - a multi-modal marker and mediator in atherosclerotic vascular disease. Atherosclerosis 236(2), 327-337 (2014).

6 Hamias R, Volvich L, Paran E et al. Low osteopontin n-terminal fragment and carotid plaque stability associated with statin or antiplatelet therapy. Curr. Vasc. Pharmacol. 14(3), 288-294 (2016).

7 Carbone F, Rigamonti F, Burger F et al. Serum levels of osteopontin predict major adverse cardiovascular events in patients with severe carotid artery stenosis. Int. J. Cardiol. 255, 195-199 (2018).

8 Zwakenberg SR, Van Der Schouw YT, Schalkwijk CG, Spijkerman AMW, Beulens JWJ. Bone markers and cardiovascular risk in Type 2 diabetes patients. Cardiovasc. Diabetol. 17(1), 45 (2018).

9 Gomez-Ambrosi J, Catalan V, Ramirez B et al. Plasma osteopontin levels and expression in adipose tissue are increased in obesity. J. Clin. Endocrinol. Metab. 92(9), 3719-3727 (2007).

10 Sawaki D, Czibik G, Pini M et al. Visceral adipose tissue drives cardiac aging through modulation of fibroblast senescence by osteopontin production. Circulation doi:10.1161/CIRCULATIONAHA.117.031358 (2018)(Epub ahead of print).

11 Sabater M, Moreno-Navarrete JM, Ortega FJ et al. Circulating pigment epithelium-derived factor levels are associated with insulin resistance and decrease after weight loss. J. Clin. Endocrinol. Metab. 95(10), 4720-4728 (2010).

12 Moreno-Navarrete JM, Ortega F, Serino M et al. Circulating lipopolysaccharide-binding protein (LBP) as a marker of obesity-related insulin resistance. Int. J. Obes. 36(11), 1442-1449 (2012).

13 Zhao G, Hu M, Li C et al. Osteopontin contributes to effective neutrophil recruitment, IL-1beta production and apoptosis in Aspergillus fumigatus keratitis. Immunol. Cell. Biol. 96(4), 401-412 (2018).

$14 \mathrm{Ge}$ Q, Ruan CC, Ma Y et al. Osteopontin regulates macrophage activation and osteoclast formation in hypertensive patients with vascular calcification. Sci. Rep. 7, 40253 (2017).

15 Hag AM, Pedersen SF, Christoffersen C et al. (18)F-FDG PET imaging of murine atherosclerosis: association with gene expression of key molecular markers. PLoS ONE 7(11), e50908 (2012).

16 Qiao H, Wang Y, Zhang R et al. MRI/optical dual-modality imaging of vulnerable atherosclerotic plaque with an osteopontin-targeted probe based on Fe3O4 nanoparticles. Biomaterials 112, 336-345 (2017).

17 Hernandez P, Whitty C, John Wardale R, Henson FM. New insights into the location and form of sclerostin. Biochem. Biophys. Res. Commun. 446(4), 1108-1113 (2014).

18 Gaudio A, Privitera F, Pulvirenti I, Canzonieri E, Rapisarda R, Fiore CE. The relationship between inhibitors of the Wnt signalling pathway (sclerostin and Dickkopf-1) and carotid intima-media thickness in postmenopausal women with Type 2 diabetes mellitus. Diab. Vasc. Dis. Res. 11(1), 48-52 (2014).

19 Gaudio A, Fiore V, Rapisarda R et al. Sclerostin is a possible candidate marker of arterial stiffness: results from a cohort study in Catania. Mol. Med. Rep. 15(5), 3420-3424 (2017).

20 Morales-Santana S, Garcia-Fontana B, Garcia-Martin A et al. Atherosclerotic disease in Type 2 diabetes is associated with an increase in sclerostin levels. Diabetes Care 36(6), 1667-1674 (2013).

21 He XW, Wang E, Bao YY et al. High serum levels of sclerostin and Dickkopf-1 are associated with acute ischaemic stroke. Atherosclerosis 253, 22-28 (2016).

22 Krishna SM, Seto SW, Jose RJ et al. Wnt signaling pathway inhibitor sclerostin inhibits angiotensin ii-induced aortic aneurysm and atherosclerosis. Arterioscler. Thromb. Vasc. Biol. 37(3), 553-566 (2017).

23 Badimon L, Borrell-Pages M. Wnt signaling in the vessel wall. Curr. Opin. Hematol. 24(3), 230-239 (2017).

24 Fruhbeck G. Bariatric and metabolic surgery: a shift in eligibility and success criteria. Nat. Rev. Endocrinol. 11(8), $465-477$ (2015). 
